\title{
Power Coefficient Analysis of Double-blade Half-rotating Impeller Tidal Turbine Operating at Yaw
}

\author{
Cong Liu ${ }^{1}$, Xiaoyi Wang ${ }^{1, *}$, Xiaofeng Yu ${ }^{1}$, Yuhua Zhang ${ }^{1}$, Zhizhen Qiu $^{1}$, Xiangrong Xu ${ }^{1}$ \\ ${ }^{1}$ School of mechanical engineering, Anhui University of Technology, Ma' anshan 243002, Anhui Province, China
}

\begin{abstract}
The double-blade half-rotating impeller tidal turbine (DHITT) is a new type of vertical shaft tidal current turbine with lift and resistance performance. The power coefficient of the DHITT is affected by the flow direction. In order to research the power coefficient $\left(C_{\mathrm{P}}\right)$ of the DHITT under different flow direction, the optimal attack flow angle of a half-impeller turbine was explored, and the fluctuation of power coefficient of the DHITT operating at yaw was analyzed based on the optimal attack flow angle. The unsteady flow of the turbine was simulated by overlapping grid technique, and the fluctuation of the turbine's power coefficient under different flow directions was analyzed, which was verified by experiments. The results have demonstrated that the power coefficient at the optimal angle of attack is 0.53 . As the yaw angle greater than $30^{\circ}$, the power reduction is nearly $40 \%$, but the average efficiency loss is only $3.7 \%$ in the range of $-3^{\circ}$ to $3^{\circ}$.
\end{abstract}

\section{Introduction}

Tidal current has the advantages of high energy density, strong regularity and sustainability, which has been concerned by a sea of domestic and foreign scientific research groups [1-2]. Water turbine is a energy conversion device, which is applied in the complex Marine environment. its performance will be affected by many factors, such as the flow direction which is mainly due to the topographic conditions and Coriolis force (earth rotation deflection force) combined effect [3]. The influence of the flow direction on turbine performance has been studied deeply by scholars at home and abroad.

Wang analyzed the influence of yaw for the average energy utilization rate of a vertical axis turbine with fixed deflection angle, and found that it is hardly unaffected under yaw condition [4]. Through CFD simulation and experiment, Wang found that the power coefficient of the horizontal axis turbine decreases with the increase of the yaw angle [5]. Fergal verified that the efficiency of the horizontal axis turbine decreases significantly when the yaw angle is greater than $15^{\circ}$ by simulation [6]. Pascal found through experiments that the power of the horizontal axis tidal current turbine was reduced by $20 \%$ when yaw was $22.5^{\circ}$ [7]. Xu analyzed the influence of frequency difference in yaw for the performance and trailing vortex of the vertical-axis turbine with variable deflection angle [8]. Liu, based on overlapping grids, studied the dynamic yaw response of a turbine and the turbine efficiency fluctuation under different yaw angles, and explored the influence of tail fins of different areas on the flow performance of a turbine [9].Wang analyzed the influence of pitch angle on the speed and load of the horizontal shaft turbine, and found that the optimal speed decreased with the increase of pitch angle, but the maximum load is less affected by the pitch angle[10].

For the asymmetry of the motion of the DHITT [11], its performance, compared with traditional vertical axis tidal turbine, is significantly different under yaw condition. The CFD numerical simulation was adopted in this study for exploring the power coefficient of the DHITT under different flow directions, to find the optimal attack flow angle of the HRITT and to provide a theoretical basis for the design of yaw mechanism. Ultimately, the results were confirmed by experiments. As a new type of vertical shaft tidal current turbine with lift and resistance, the DHITT has the advantages of higher power coefficient and better starting performance. Therefore, it is of value and significance to study the effect of yaw on its power coefficient.

\section{Working principle of the DHITT}

The DHITT is evolved from the half-rotating mechanism (HRM), which can be realized by sprocket/belt wheel or planetary gear.

As shown in Fig. 1, the ratio of diameter of fixed gear and driving wheel is 1:2. When the rotating arm at angular velocity, $\omega$, a half-rotating component which is fixedly connected with the driving wheel rotates in the same direction as $\omega / 2$. Thus, when the rotating arm rotates once, the half-rotating component rotates only half turn, which leads to asymmetric motion, so it is called "half-rotating mechanism".

\footnotetext{
* Corresponding author: wangxy@ahut.edu.cn
} 


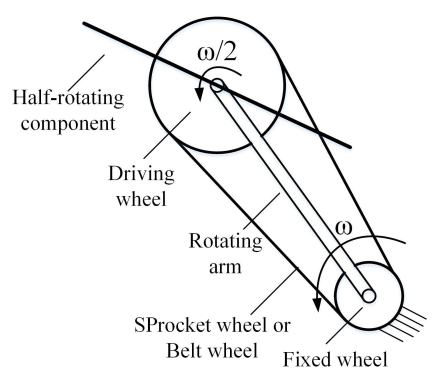

(a) Sprocket/ belt transmission

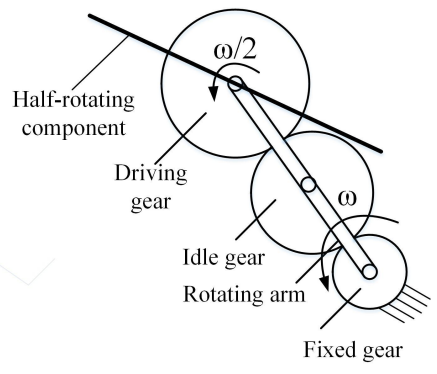

(b) Planetary gear transmission

Fig. 1. Transmission mode of the HRM

Through the deformation design and derivative design of the structural form of the HTM, as shown in Fig. 2, two driving gears are installed at both ends of the rotating arm respectively, at the same time, the driving wheel is fixed on the blade, thus constituting the DHITT.
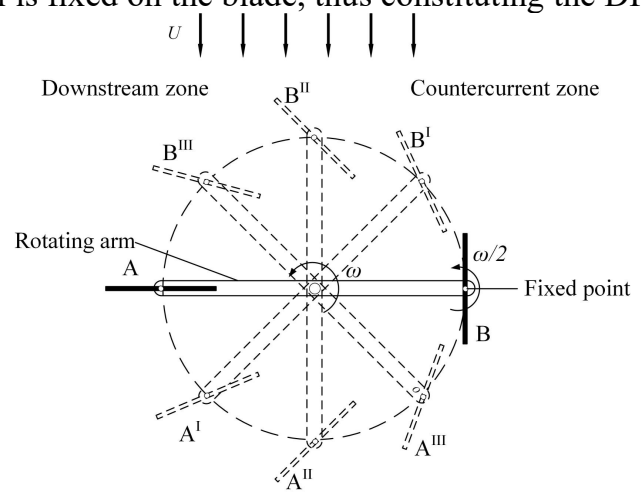

Fig. 2. Working principle of the DHITT

Based on the characteristics of the HTM motion, there is a fixed point on the half-rotating blade. In the process of the blade movement, blade itself (or blade extension line) always passes through the fixed point. Thus, its position can be determined that when the rotating arm is at different positions, the position of the blade is shown in Fig. 2. Blade A and blade B represent the initial position of the turbine blade. The blade AI, blade BI, blade AII, blade BII, blade AIII and blade BIII respectively represent the phase of the blade when the rotating arm rotates at different positions.

For the influence analysis of water flow direction on the energy of the DHITT, the following concepts are proposed:

i. Define the initial position of the DHITT: In the motion law of turbine blades, the position where one blade is collinear with the rotating arm and the other blade is perpendicular to the rotating arm ,so it was called the initial position.

ii. Define a non-yaw state:During the law of turbine blade movement, when the flow direction is perpendicular to the initial position of the rotating arm, it was defined as the non-yaw state.

\section{Modeling of yaw calculation for the DHITT}

\subsection{Operating principle of a half-impeller turbine at yaw}

According to wind turbine research, the yaw of a wind turbine means that the wind turbine rotates in the vertical direction or the direction of the tower, so as to respond to the change of the incoming wind direction [12-13].Thus, the concept of yaw was introduced. The included angle between the flow direction and the normal of the initial phase of rotating arm was defined as the yaw angle. When the included angle is zero degree, that is non-yaw state. (The left incoming flow was defined as negative yaw and the right incoming flow was defined as positive yaw in Fig.3.). The incoming flow direction at positive or negative yaw, $\pm \alpha$, is shown in Fig. 3 .

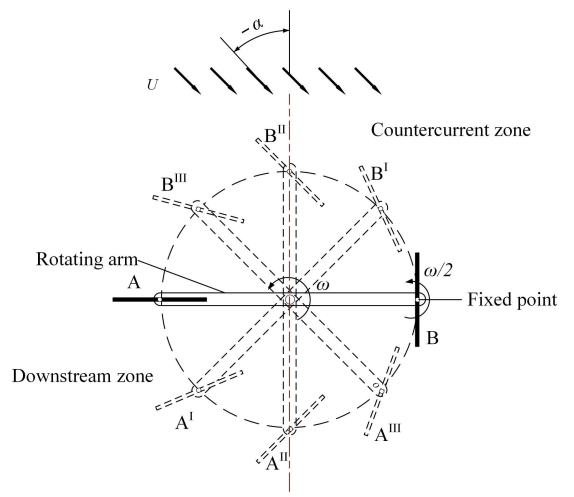

(a) Negative yaw $(-\alpha)$

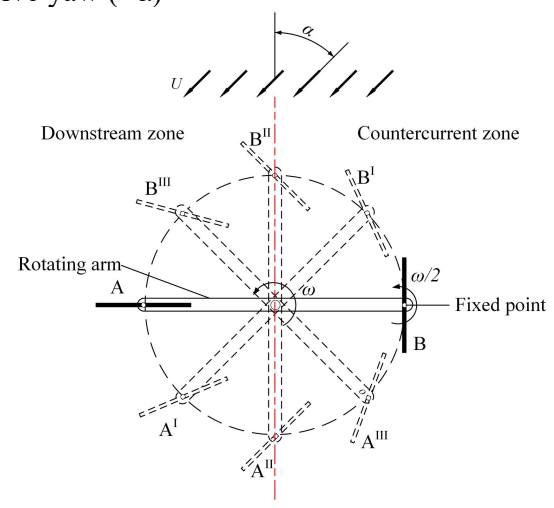

(b) Positive yaw $(+\alpha)$

Fig. 3. Yaw condition

Under the condition that the incoming flow direction is unchanged, the incoming flow direction can be changed through the phase of the offset turbine. As shown in Fig. 4 , the angle of clockwise rotation of the turbine, $\alpha$, is equivalent to negative yaw, $-\alpha$, and similarly, the angle of counterclockwise rotation of the turbine, $\alpha$, is equivalent to positive yaw, $+\alpha$. 


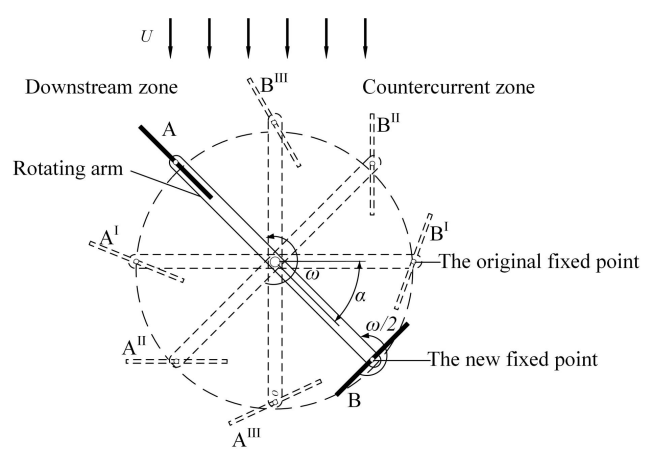

(a) Angle of clockwise rotation of the turbine $(-\alpha)$
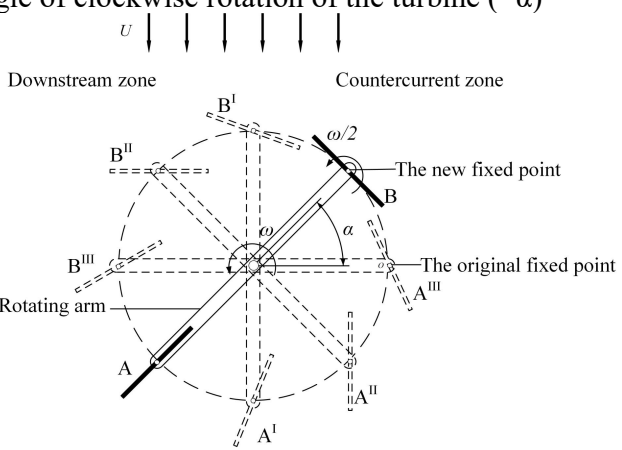

(b) Angle of counterclockwise rotation of the turbine $(+\alpha)$ Fig. 4. Yaw of a water turbine

\subsection{Model of the DHITT}

\subsubsection{Structural parameters and CFD calculation model of the DHITT}

According to the previous experimental study of our research group [14], the size parameters of the DHITT is presented in Table1.

Table 1. Structural parameters of the DHITT

\begin{tabular}{cc}
\hline Structure parameters & Value \\
\hline Chord length of blade ( $\mathrm{a})$ & $0.2 \mathrm{~m}$ \\
Blade height ( $\mathrm{h})$ & $0.9 \mathrm{~m}$ \\
Rotating arm radius ( $\mathrm{R})$ & $0.085 \mathrm{~m}$ \\
Blade number ( N ) & 2 \\
\hline
\end{tabular}

The $3 \mathrm{D}$ (three dimensional)model of a DHITT is shown in Figure 5:

In this paper, the yaw state of the DHITT was numerically simulated based on overlapping grids with CFD software and verified by experiments. Background grid and overlapping grid area are established in Fig. 6. Due to blade motion includes both rotation and revolution, the UDF was adopted to define blade motion. For the fluid medium is water, the viscosity of the fluid medium was ignored, so inviscid model was used for calculation. The blade of the turbine is normally a flat blade, the influence of spread length can be ignored. Therefore, two-dimensional numerical simulation was adopted. In order to improve the accuracy of data, the number of grids in moving area was encrypted. At the same time, the background grid was rotated to simulate different flow directions under the same mesh size.

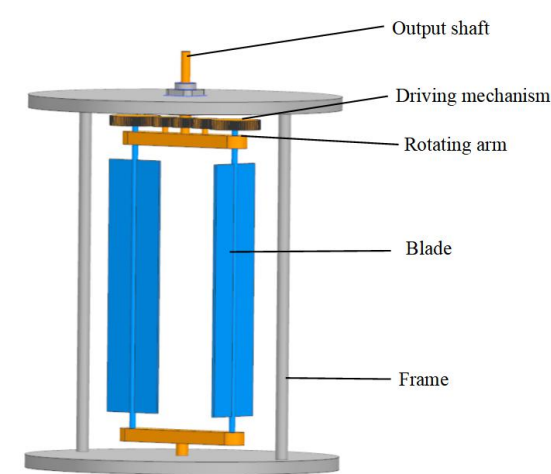

Fig. 5. 3D model of the DHITT

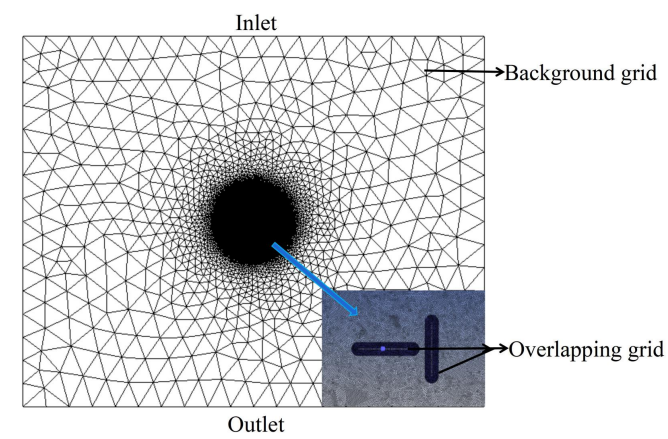

(a) Boundary conditions

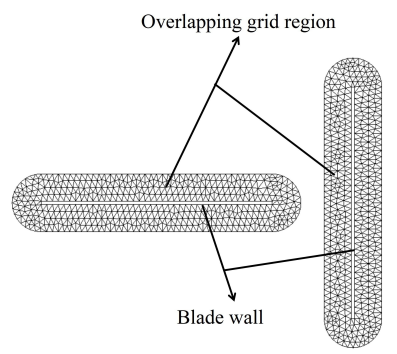

(b) Blade boundary

Fig. 6. Calculation model

\subsubsection{Yaw calculation model of the DHITT}

Taking positive yaw, $+\alpha$, as an example, A yaw calculation model was established. An inertial coordinate system, Zxy, as shown in Fig.7, is established at a vertical position of the rotating arm and the direction of incoming flow. The origin point, $\mathrm{Z}$, is located at the rotary center of the rotating arm, and the x-axis passes through the rotary center of the blade. The dynamic coordinate system, $\mathrm{O} \xi \eta$, is fixed on the blade, the origin point , $\mathrm{O}$, is located in the center of the blade axis, and the $\xi$-axis is parallel to the blade. At the initial moment, the origin point of dynamic coordinate system, $\mathrm{O}$, is located on the positive half of $x$-axis, and an angle, $\alpha / 2$, is between the $\mathrm{x}$-axis and $\xi$-axis. When the rotating arm rotates one angle, $\varphi$, counterclockwise, the dynamic coordinate system rotates half an angle, $\varphi / 2$. and the position, $\mathrm{P}(0, \eta)$, at any point of the blade in the inertial coordinate system position, $\left(x_{\mathrm{p}}, y_{\mathrm{p}}\right)$, can be expressed as:

$$
\left\{\begin{array}{l}
x_{p}=R \cos \varphi-\eta_{\mathrm{p}} \sin \left(\frac{\varphi}{2}+\frac{\alpha}{2}\right) \\
y_{p}=R \sin \varphi+\eta_{\mathrm{p}} \cos \left(\frac{\varphi}{2}+\frac{\alpha}{2}\right)
\end{array}\right.
$$


The derivative of equation 1 with respect to time, $t$, gives the velocity component of the $\mathrm{P}$ in the inertial coordinate system. This can be expressed as:

$$
\left\{\begin{array}{c}
v_{x_{p}}=\frac{d x_{p}}{d t}=-\frac{d \varphi}{d t} R \sin \varphi-\frac{1}{2} \frac{d \varphi}{d t} \eta_{p} \cos \left(\frac{\varphi}{2}+\frac{\alpha}{2}\right) \\
v_{y_{p}}=\frac{d y_{p}}{d t}=\frac{d \varphi}{d t} R \cos \varphi-\frac{1}{2} \frac{d \varphi}{d t} \eta_{p} \sin \left(\frac{\varphi}{2}+\frac{\alpha}{2}\right)
\end{array}\right.
$$

The velocity component of the velocity at point, $\mathrm{P}$, in the dynamic coordinate system can be expressed as:

$$
\left\{\begin{array}{l}
v_{\xi_{p}}=v_{x_{p}} \cos \left(\frac{\varphi}{2}+\frac{\alpha}{2}\right)+v_{y_{p}} \sin \left(\frac{\varphi}{2}+\frac{\alpha}{2}\right) \\
v_{\eta_{p}}=v_{y_{p}} \cos \left(\frac{\varphi}{2}+\frac{\alpha}{2}\right)-v_{x_{p}} \sin \left(\frac{\varphi}{2}+\frac{\alpha}{2}\right)
\end{array}\right.
$$

Therefore, the direction of water flow is always parallel to y-axis of the inertial coordinate system. The velocity, U,can be expressed as the velocity component of the dynamic system:

$$
\left\{\begin{array}{l}
v_{\xi_{U}}=U \sin \left(\frac{\varphi}{2}+\frac{\alpha}{2}\right) \\
v_{\eta_{U}}=U \cos \left(\frac{\varphi}{2}+\frac{\alpha}{2}\right)
\end{array}\right.
$$

The velocity component of the relative velocity in the dynamic coordinate system can be expressed as:

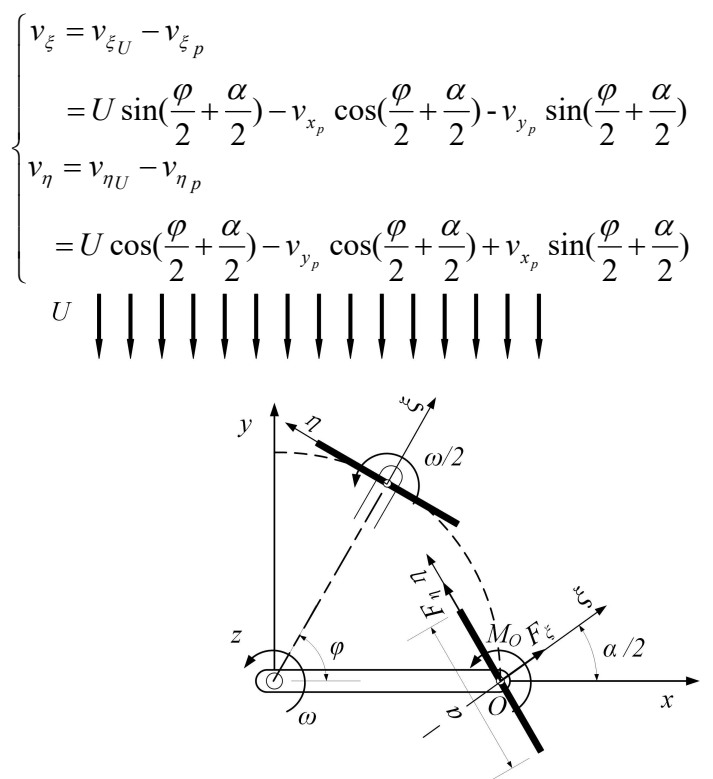

Fig. 7. Positive yaw calculation mode

Where, $\omega$ is the rotating speed of the tumbler, $\alpha$ is the deflection angle of the flow.

In formula (6), adding half of an angle, $\alpha / 2$, represents the whole Angle of positive yaw, $+\alpha($ Positive yaw, $+\alpha$, range $0^{\circ}$ to $+90^{\circ}$ ), and half of an angle, $-\alpha / 2$, represents the whole Angle of negative yaw, $-\alpha$ (negative yaw, $-\alpha$, range $0^{\circ}$ to $-90^{\circ}$ ). When the inflow is non-yaw condition, the $\alpha$ is $0^{\circ}$. When $\alpha$ exceeds $90^{\circ}$ or $-90^{\circ}$, the turbine reverses.

The $v_{\xi}$ is the relative velocity on the vertical blade surface, which determines the normal force exerted by the fluid on the blade.The $v_{\xi}$ at the point on the blade varies with the $\mathrm{P}(0, \eta)$ and the $\varphi$. The pressure difference on the blade surface pushes the arm to rotate, and the fluid pressure on the blade is not equal. Therefore, there is also its own torque, $M$, on the blade axis. The $v_{\eta}$ is the relative velocity parallel to the chord surface of the blade, whose magnitude determines the force exerted by the fluid on the blade side.

\subsubsection{Blade hydrodynamic calculation}

For the flat blade, the hydrodynamic force received by the single blade was calculated,which is relevantly the function of the velocity components $v_{\xi}$ and $v_{\eta}$. The hydrodynamic force received by the blade was simplified as the force and moment on the blade spindle, and is given by:

$$
\left\{\begin{array}{c}
F_{\xi}=\frac{1}{2} C_{\mathrm{d}} \rho \mathrm{h} \frac{\left(\int_{-\frac{a}{2}}^{\frac{a}{2}} v_{\xi} d \eta\right)^{2}}{a} \\
F_{\eta}=\frac{1}{2} C_{\mathrm{d}} \rho \mathrm{h} \frac{\left(\int_{-\frac{a}{2}}^{\frac{a}{2}} v_{\eta} d \eta\right)^{2}}{b} \\
M_{o}=\int_{0}^{\frac{a}{2}} \frac{1}{2} C_{d} \rho h v_{\xi} \eta d \eta-\int_{-\frac{a}{2}}^{0} \frac{1}{2} C_{d} \rho h v_{\xi} \eta d \eta
\end{array}\right.
$$

Where, $\mathrm{H}$ is blade length, $\mathrm{a}$ is the chord length of the blade, $\mathrm{b}$ is blade thickness, $C_{\mathrm{d}}$ is the fluid resistance coefficient of the blade, $F_{\xi}$ is the component force along the $\xi$-axis, $F_{\eta}$ is the component force along the $\eta$-axis, Mo is the moment of the $O$.

The positions of forces and torque acting on the main shaft of the blade are at the intersection of the main shaft of the blade and the rotating arm, which was converted into the torque towards the arm. Therefore, the output torque of a single blade pushing the turbine can be expressed as:

$$
M_{\text {single }}(\omega, \varphi)=F_{\xi} * R * \sin \left(\frac{\varphi-\alpha}{2}\right)+\frac{1}{2} M_{o}-F_{\eta} * R * \cos \left(\frac{\varphi-\alpha}{2}\right)
$$

Where, $\mathrm{R}$ is the radius of the arm

The phase difference, $\pi$, of the double-blade turbine blade, Therefore, the total output torque of the turbine can be expressed as:

$$
M=M(\omega, \varphi)+M(\omega, \varphi+\pi)
$$

Assuming that in the uniform incoming flow, $U$, the turbine will rotate around its center at constant angular velocity, $\omega$. The power coefficient of the DHITT can be defined as:

$$
C_{\mathrm{p}}=\frac{M \omega}{\frac{1}{2} \rho S U^{3}}
$$

Where, $\rho$ is the density of the fluid, $\mathrm{S}$ is the swept area of the turbine, $U$ is the incoming flow velocity. 


\section{प्The optimal attack flow angle of DHITT}

In order to explore the turbine's energy efficiency under yaw condition, the non-yaw state was defined as the yaw angle of $0^{\circ}$. The optimal power coefficient of the turbine under different inlet angles was obtained through CFD numerical simulation, as shown in Fig. 8.

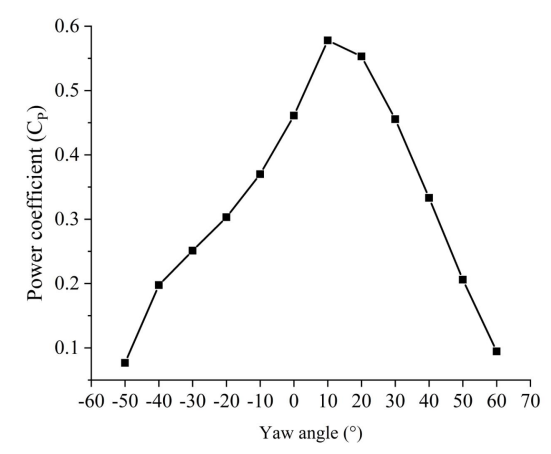

Fig. 8. Power coefficient curves

The yaw angle under the highest power coefficient was taken as the optimal attack flow angle of the DHITT. Fig.10 displays the highest power coefficient is 0.575 for positive yaw of $10^{\circ}$. Taking the positive yaw of $10^{\circ}$ as the reference, by changing the blade installation phase, the installation phase was defined as the optimal incoming flow phase. The main reason for the improvement of power coefficient of positive yaw at $10^{\circ}$ was that small angle yaw reduced the blocking effect of water flow on the blades in the countercurrent zone. During the process of rotation, there was a certain proportion of overlap area of the DHITT ( the overlap area of the two blades on the normal projection along the direction of the inflow), which can effectively reduce or eliminate, to some extent, the negative moment generated in the process of impeller rotation. In the flow field, with the overlapping area turbines, the resistance of the downstream was reduced by the blades the upstream blades. Through the simulation results demonstrated that the overlapping area had the best effect under the condition of positive yaw of $10^{\circ}$ of the DHITT.

\section{(1) Power coefficient analysis of the turbine under yaw condition}

The optimal attack flow angle was taken as new nonyaw state to analyze the influence of yaw condition on the power coefficient of the DHITT. The CFD numerical simulation was conducted to analyze the power coefficient curve of yaw condition as shown in Fig. 9.

Based on the optimal angle of attack, the effect of yaw on the power coefficient of the DHRITT was analyzed. It is shown that the yaw angle of $0^{\circ}$ turbine capacity coefficient is 0.57 in Fig. 9(a). However, the power coefficient of the turbine at $30^{\circ}$ negative yaw is 0.30 , and the turbine power coefficient at $30^{\circ}$ positive yaw is 0.33 . When the yaw angle was greater than $30^{\circ}$, the power reduction of the turbine was about $45 \%$.
Although the DHITT is a vertical axis turbine, it has higher energy acquisition efficiency compared with the same type of vertical axis turbine. Compared with the horizontal axis turbine, it is also affected by the direction of incoming flow. The smaller the angle between the direction of incoming flow and the initial position of the arm, the lower the efficiency.This means that the DHITT need yaw mechanisms to enable it to be used over a wider area of water. For the design of yaw mechanism, a higher yaw response was required when the yaw angle is greater than $30^{\circ}$ to reduce the influence of flow deflection for the energy acquisition coefficient. It should be noted that when the yaw angle is between $-3^{\circ}$ and $3^{\circ}$, the average power reduction of the DHITT is only $3.7 \%$ in Fig. 9(b).

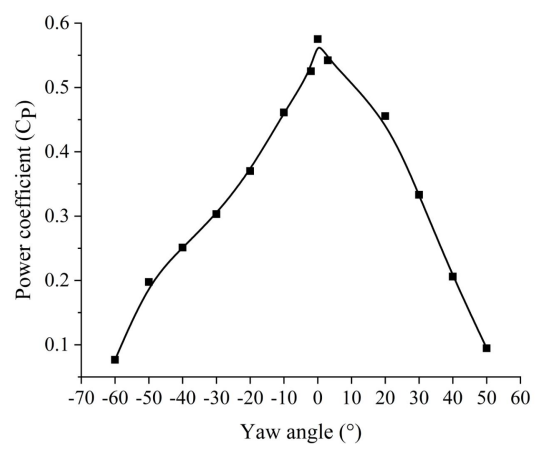

(a) Large angle yaw curve

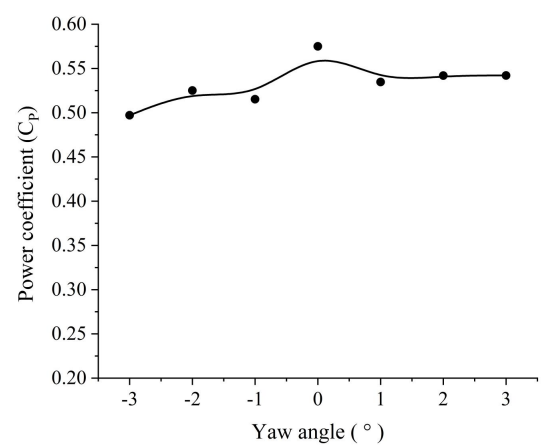

(b) Small angle yaw curve

Fig. 9. Energy acquisition curve under yaw condition

Therefore, the fluctuation of small-angle yaw between $-3^{\circ}$ and $3^{\circ}$ can ignore, which will provide a basis for the design of the yaw mechanism of the double-blade half-rotating impeller turbine.By analyzing the efficiency fluctuation of the DHITT, it can be known that the allowable yaw angle of the yaw mechanism is $3^{\circ}$.In other words, under the action of yaw mechanism, the allowable angle fluctuation range from $-3^{\circ}$ to $3^{\circ}$ after the turbine returns to the optimal angle of attack.

\section{Experimental verification and result analysis}

This experiment was conducted in open waters which is clam. The propeller was used to push the experimental platform to simulate the flow, and a velocity was measured by ADV (Acoustic Doppler 
Velocimetry) current meter. A experimental prototype was equipped with the senor to measure rotor torque and rate of rotation. The experimental environment is shown in Fig. 10. The horizontal operation of the experimental platform can realize different flow directions by changing the installation phase of turbine blades.

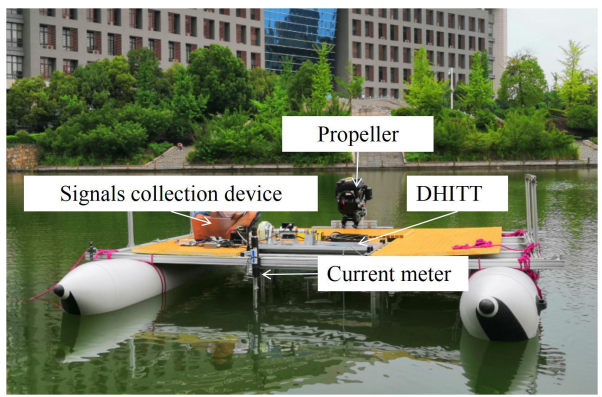

(a) Experimental platform

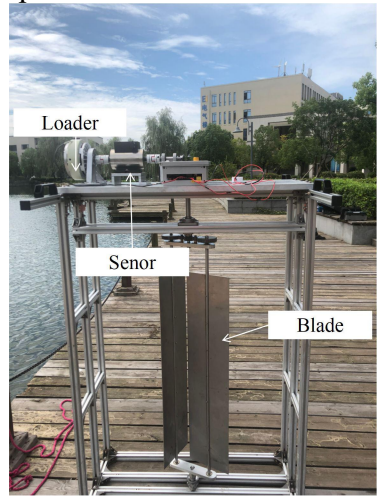

(b) Experimental prototype

Fig. 10. Underwater Experiment

The experimental platform operated at a speed of $0.8 \mathrm{~m} / \mathrm{s}$. The influence of different flow directions on the turbine's power coefficient under the same speed was analyzed, and the optimal attack angle of the halfimpeller turbine was explored. Experimental tests were conducted include a non-yaw condition $\left(\alpha=0^{\circ}\right)$ and yaw conditions $\left(\alpha=10^{\circ}, 20^{\circ}, 40^{\circ},-20^{\circ},-40^{\circ}\right)$, respectively, and the energy acquisition curve shown in Fig. 11.

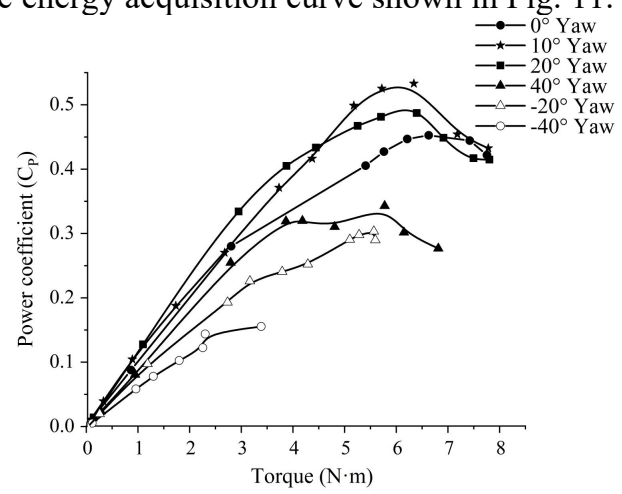

Fig. 11. Energy efficiency of a half-impeller turbine under different inflow directions

The experimental curve indicates that an optimal load is corresponding to different yaw angles, so that the turbine's power coefficient is optimal. From the experimental results, it can be concluded that the maximum energy point is $10^{\circ}$ positive yaw, and its power coefficient is 0.53 , which is consistent with the simulation prediction of the best attack flow angle. The optimal energy acquisition points at different yaw angles in the experiment were compared with those obtained by CFD numerical simulation at different yaw angles, as shown in Fig. 12.

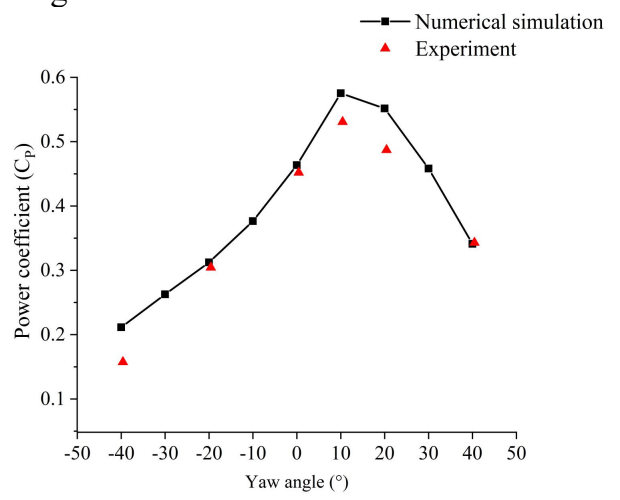

Fig. 12. Variation curve of the power coefficient

The main reasons for the error were that the overlapping grids was used, which is a certain error in the numerical mapping, and the unit growth of twodimensional numerical simulation was calculated, while the $0.9 \mathrm{~m}$ blade was used in the experiment. The maximum relative error between the numerical simulation results and the experimental results does not exceed $6.58 \%$, and the average relative error is $2.89 \%$. The numerical results were slightly higher than the experimental results. Although the simulation results will have some error, the overall trend was consistent. The optimal energy gain coefficient at $10^{\circ}$ positive yaw is 0.53 , and $10^{\circ}$ positive yaw was taken as the optimal attack flow angle (i.e,the new non-yaw state), as shown in Fig. 13.

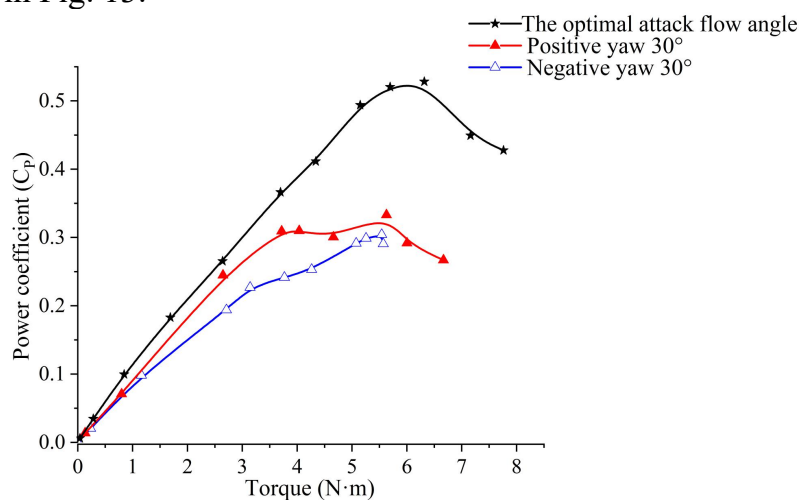

Fig. 13. Energy acquisition curve under yaw condition

The experimental results showed that the $C_{\mathrm{P}}$ is 0.302 at $30^{\circ}$ negative yaw and 0.343 at $30^{\circ}$ positive yaw. Compared with the optimal energy gain coefficient of 0.53 at the optimal angle of attack, the power reduction loss is $43.2 \%$ and $35.8 \%$ respectively. Under the condition of the same flow velocity, the peak load at the optimal angle of attack is higher than the peak load at the yaw state, which is mainly caused by the motion asymmetry of the DHITT. In the operation cycle of the two blades, under yaw state, when the downstream blade was doing positive work, the negative work interval of 
the upstream blade was increasing, resulting in the decrease of peak load The smaller the angle between the incoming flow direction and the tumbler at the initial position, the larger the area of negative work of the blades.

\section{Conclusion}

Based on CFD simulation software, this paper analyzed the hydrodynamic performance under the yaw state of the DHITT with the specific structure size, and verified it through experiments. Meanwhile, it provided the basis for the design of the yaw mechanism of the DHITT.

(1) Based on the non-yaw state, the DHITT exited an optimal angle of attack, and the energy coefficient of the optimal angle of attack was 0.53 .

(2)The optimal attack flow angle was regarded as the new non-yaw state. The average efficiency loss of DHRITT at a small yaw angle of $-3^{\circ}$ to $3^{\circ}$ was 0.0377 . The yaw of the small angle had little influence on the energy coefficient of the double-blade half-rotating impeller turbine.

(3) By analyzing the $C_{\mathrm{P}}$ of the DHITT under yaw condition, it was found that the flow direction had a great influence on the energy acquisition efficiency of DHITT after the yaw angle was greater than $10^{\circ}$. The experimental results show that the power reduction was $43.2 \%$ for negative yaw at $30^{\circ}$ and $35.8 \%$ for positive yaw at $30^{\circ}$.

In this paper, the research on the $C_{\mathrm{P}}$ of the DHITT operating at yaw will provide theoretical guidance for the design of a specific yaw device for the DHITT, but this paper lacks the research on the power stability of the DHITT.

This work is supported by the Key R \& D Program of Anhui, China (Grant No.201904b11020030).

\section{References}

1. S M Wang, M M Li,Z Y LI, K TIAN.Ship Engineering, Development overview of international tidal energy utilization technology. 42 (2020).

2. W M Liu,C L Ma,F Y Chen,L Liu, Y Z Ge , J P Peng, H Y Wu, Q B Wang. Advances in marine science, Development and Utilization of Marine renewable energy and technological progress. $36,1671-6647(2018)$.

3. L Zhang, J H Shang, Z Y Zhang, J Jiang, X H Wang. Journal of hydraulic power generation, Research status of tidal current energy 2015 hydrodynamics. 35, 1-15(2016).

4. K Wang, K Sun, L Zhang, Q H Sheng, X W Zhang. Journal of Shanghai jiao tong university, Effect of bow sway on hydrodynamic performance of vertical shaft tidal turbine. 50, 563-568+574(2016).

5. S J Wang, X Y Jiang, P Yuan, X C Si, X L Yu. Journal of ocean university of China, Analysis on performance and wake field characteristics of tidal turbine under yaw condition. 49, 122-133(2019).
6. F Rourke, F Boyle, A Reynolds, D Kennedy. Energy, Hydrodynamic performance prediction of a tidal current turbine operating in non-uniform inflow conditions. 93, 2483-2496(2015).

7. P W Galloway, L E Myers, A S Bahaj. Marine and ocean technology, Experimental and numerical results of rotor power and thrust of a tidal turbine operating at yaw and in waves. 2246-2253(2011).

8. G Xu, S Q Wang, R Q Zhu, L Zhang. Journal of Harbin Engineering University, Hydrodynamic Analysis of Bow Motion of a Vertical turbine with variable deflection angle. 39, 304-309(2012).

9. Y Liu, W J Cai, J B Yi. Ocean Engineering Equipment and Technology, Research on dynamic Yaw Hydrodynamic Characteristics of Hydraulic Turbines based on Overlapping grids. 5, 3439(2015).

10. B Z Wang, $\mathrm{T} \mathrm{Y}$ Hu, Y Teng, Y Guo, Y F Zhang. China Ocean Engineering, Research on Pitch Control Strategies of Horizontal Axis Tidal Current Turbine. 34, 223-231(2020).

11. H Qiu, X Y Wang,F Q Chen,Z Z Qiu. Mechanical design, Research on the minimization design of halfrotating mechanism. 34, 75-80(2017).

12. D Wood. Small wind turbines:analysis,design and application.( Springer-Verlag,2011).

13. A Saleem, M H Kim. Energy, Performance of buoyant shell horizontal axis wind turbine under fluctuating yaw angles. 169, 79-91(2019).

14. X Y Wang, L Li, K Xue. ICNERA, Experimental Research on Energy Extraction Characteristics for Half-rotating Impeller Tidal Turbine. 7, 193199(2020). 